June 22, $2021 \quad 7: 47$ WSPC/INSTRUCTION FILE

Article'Rybalov ${ }^{\circ}$ Osetrin 'Makarenkov

Modern Physics Letters A

(C) World Scientific Publishing Company

\title{
COSMOLOGICAL MODELS WITH THE SPINOR AND NON-MINIMALLY INTERACTING SCALAR FIELD
}

\author{
Osetrin K.E.* \\ Makarenko A.N. ${ }^{\dagger}$ \\ Tomsk State Pedagogical University, \\ Tomsk, Russian Federation \\ Rybalov Yu.A. ${ }^{\ddagger}$ \\ Tomsk State Pedagogical University, \\ Tomsk, Russian Federation, \\ National Research Tomsk State University, \\ Tomsk, Russian Federation \\ Received (Day Month Year) \\ Revised (Day Month Year)
}

\begin{abstract}
The solution to the current extending Universe problem, and the description of all stages of evolution compels scientists to consider various cosmological models. Scalar tensor models are rather simple and also allow us to clearly define the separate stages of evolution. Furthermore, other cosmological models are reduced. Our work takes into consideration the non-minimally interacted scalar field and the spinor field. The spinor field has been considered to establish a better understanding of the stages of evolution in our Universe.
\end{abstract}

Keywords: cosmological model; non-minimally interactions; scalar field.

PACS Nos.: 04.50.Kd, 95.36.+x, 98.80.-k, 98.80.Cq

\section{Introduction}

The problem of dark energy and dark matter is one of the main challenges in modern cosmology. Astrophysical data indicates that the observed universe is in an accelerated phase. This acceleration could be caused by this so-called dark energy (see Refs. 1, 2, 3, 4, 5, 6, 7, 8, 9, 10, 11, 12 for a recent review). These discoveries have set new serious challenges for theoretical physics and have prompted speculation, mostly based on phenomenological ideas which involve new dynamical sources of gravity that act as dark energy, and/or various modifications to general

\footnotetext{
*osetrin@tspu.edu.ru

†andre@tspu.edu.ru

${ }^{\ddagger}$ ribalovyua@tspu.edu.ru
} 
relativity. The spectrum of models, having been postulated and explored in recent years, is extremely wide and includes, in particular, Quintessence, K-essence, Ghost Condensates, Dvali-Gabadadze-Porrati gravity, Galileon gravity, and $f(R)$ gravity Refs. 13, 14, 15, 16 (see Refs. 17 for detailed reviews of these and other models). Such cosmological models tend to describe not only the accelerated expansion at this stage of evolution, but also at all other stages of evolution. All these models are divided into two main classes: $f(R)$ theory and alike (see Refs. 13, 17, 18, 19, 20), and models which use various objects: scalars, spinor, cosmological constant, liquid with the difficult state equation.

The spinor fields have been used as a source of a gravitational field in a number of works, and the interaction of the spinor and scalar fields as a factor of inflation expansion has been considered. Friedman-Robertson-Walker's (FRW) approach to the real space-time is considered to be the closest, at the same time the estimated change of a metrics conformal factor for the analysis of behavior of the Universe in cosmological models is considered the most often. In this work we will study cosmological models similar to those offered in the works Refs. 21, 22, but with nonminimally interacted scalar field. Most of the phenomenological models represent various modifications of scalar-tensor theories. The models allowing non-minimal interaction of scalar field derivatives and curvature are of particular interest. As Amendola showed, the theory of such kind, cannot be brought to a form of Einstein gravitation by conformal transformation. Note that usually field equations in models with non-minimal derivative interaction are the differential equations above the second order. However, the order goes down to the second in a special case when the kinetic term is connected only to Einstein's tensor, i.e. $\kappa G_{\mu \nu} \phi^{\mu} \phi^{\nu}$ (see, for example Ref. 23). In works Refs. 24, 25 authors have studied cosmological scenarios from non-minimal interaction of the derivative $\kappa G_{\mu \nu} \phi^{\mu} \phi^{\nu}$, concentrating on models with zero and constant potential. According to the parameter choices, we have obtained a variety of behaviors including the Big Bang, an expanding universe without a beginning, a cosmological turnaround, an eternally contracting universe, a Big Crunch, and a cosmological bounce. In this cosmological model The nonminimal interaction of gravitation and a matter (a scalar field) is considered in a combination with the spinor field. In introduction the relevance of the considered model is studied. In section 2 the task is formulated and the main equations are given. In section 3 we will obtain the FRW equations. In section 4 we will calculate solutions and conduct asymptotic research solutions. In section 5, the results of our research will be discussed.

\section{The field equations}

Let us consider a model with the action in the form:

$$
S=\int d^{4} x \sqrt{-g}\left\{\frac{R}{8 \pi}-\left(g_{\mu \nu}+\kappa\left(R_{\mu \nu}-\frac{1}{2} R g_{\mu \nu}\right)\right) \nabla^{\mu} \phi \nabla^{\nu} \phi-V(\phi)-L_{D}\right\},
$$

where $R$ is scalar curvature. 
The Dirac Lagrangian $L_{D}$ of fermion mass field $m_{f}$ has the form:

$$
L_{D}=\frac{i}{2}\left\{\bar{\psi} \Gamma^{\mu} D_{\mu} \psi-D_{\mu} \bar{\psi} \Gamma^{\mu} \psi\right\}-m_{f}\langle\bar{\psi} \psi\rangle-F(\psi \bar{\psi}) .
$$

In the expression (2), $F(\psi \bar{\psi})$ describes the potential of fermion field and $\bar{\psi}=\psi^{\dagger} \gamma^{0}$ denotes the conjugate spinor. $\Gamma^{\mu}=e_{a}^{\mu} \gamma^{a}$ are generalized Dirac-Pauly matrices in a curved spacetime (where $e_{a}^{\mu}$ is tetrad).

A consideration of the spinor field is carried out in works Refs. 21, 22, we have used the necessary results. Let us now consider a FRW universe with the flat spatial metric:

$$
d s^{2}=-d t^{2}+a(t)^{2}\left(d x^{2}+d y^{2}+d z^{2}\right) .
$$

Einstein's equations can be written as

$$
\frac{1}{8 \pi}\left(R_{\mu \sigma}-\frac{1}{2} g_{\mu \nu} R\right)=T_{\mu \nu}
$$

where

$$
T_{\mu \nu}=-\left(T_{f}\right)_{\mu \nu}+\left(T_{\phi}\right)_{\mu \nu},
$$

$\left(T_{f}\right)_{\mu \nu}$ is the energy-momentum tensor of the fermion fields and $\left(T_{\phi}\right)_{\mu \nu}$ is the contribution of the variation of the scalar field which interacts non-minimally. A symmetric form of the energy-momentum of the fermion field is as follows:

$$
\begin{gathered}
\left(T_{f}\right)_{0}^{0}=m_{f}\langle\bar{\psi} \psi\rangle+F(\psi \bar{\psi}), \\
\left(T_{f}\right)_{i}^{i}=F(\psi \bar{\psi})-\frac{\bar{\psi}}{2} \frac{d F(\psi \bar{\psi})}{d \bar{\psi}}-\frac{d F(\psi \bar{\psi})}{d \psi} \frac{\psi}{2} .
\end{gathered}
$$

The consequence of the equations of motion of the spinor fields is given by:

$$
\frac{d}{d t} \bar{\psi} \psi+3 H \bar{\psi} \psi=0, \bar{\psi} \psi=\frac{c}{a(t)^{3}},
$$

where $H=\frac{\dot{a}}{a}$. Self-interaction potential can be written as $F=\sum_{n} \alpha_{n}(\bar{\psi} \psi)^{2 n}$, where $\alpha_{n}$ and $\mathrm{n}$ are constants.

A symmetric form of the scalar field energy-momentum tensor can be obtained from (1) in the form

$$
\begin{array}{r}
\left(T_{\phi}\right)_{\mu \nu}=\nabla_{\mu} \phi \nabla_{\nu} \phi-\frac{1}{2} g_{\mu \nu} \nabla^{\alpha} \phi \nabla_{\alpha} \phi-g_{\mu \nu} V(\phi)+\kappa\left(\frac{1}{2} \nabla_{\mu} \nabla_{\nu}\left(\nabla^{\alpha} \phi \nabla_{\alpha} \phi\right)-\right. \\
-\frac{1}{2} R \nabla_{\mu} \phi \nabla_{\nu} \phi-\frac{1}{2}\left(R_{\mu \nu}-\frac{1}{2} R g_{\mu \nu}\right) \nabla^{\alpha} \phi \nabla_{\alpha} \phi-\frac{1}{2} g_{\mu \nu} \square\left(\nabla^{\alpha} \phi \nabla_{\alpha} \phi\right)- \\
-\frac{1}{2} g_{\mu \nu} R^{\alpha \beta} \nabla_{\alpha} \phi \nabla_{\beta} \phi+2 \nabla_{\alpha} \phi \nabla_{(\mu} \phi R_{\nu)}^{\alpha}+\frac{1}{2} \square\left(\nabla_{\mu} \phi \nabla_{\nu} \phi\right)- \\
\left.-\nabla_{\alpha} \nabla_{(\mu}\left(\nabla_{\nu)} \phi \nabla^{\alpha} \phi\right)+\frac{1}{2} g_{\mu \nu} \nabla_{\alpha} \nabla_{\beta}\left(\nabla^{\alpha} \phi \nabla^{\beta} \phi\right)\right) .
\end{array}
$$

We now write the equation of motion of the scalar field as:

$$
\square \phi+\kappa \nabla_{\mu}\left(\nabla_{\nu} \phi\left(R^{\mu \nu}-\frac{1}{2} g^{\mu \nu} R\right)\right)+V^{\prime}(\phi)=0, V^{\prime}(\phi)=\frac{d V(\phi)}{d \phi} .
$$


Osetrin K.E., Makarenko A.N., Rybalov Yu.A.

\section{The field equations in the FRW}

We will choose the potential of a fermionny field $F(\bar{\psi} \psi)$ in the following form:

$$
F(\bar{\psi} \psi)=\alpha_{1}(\bar{\psi} \psi)^{2}+\alpha_{2}(\bar{\psi} \psi)^{4}+\alpha_{3}(\bar{\psi} \psi)^{6} .
$$

Einstein's equations in FRW will be as follows:

$$
\begin{gathered}
3 \dot{a}^{2}=8 \pi a^{2}\left(V(\phi)+\frac{m_{f} c}{a^{3}}+\alpha_{1} \frac{c^{2}}{a^{6}}+\alpha_{2} \frac{c^{4}}{a^{12}}+\alpha_{3} \frac{c^{6}}{a^{18}}\right)+4 \pi\left(a^{2}-9 \kappa \dot{a}^{2}\right) \dot{\phi}^{2}, \\
\dot{a}^{2}+2 a \ddot{a}=-8 \pi a^{2}\left(V(\phi)-\alpha_{1} \frac{c^{2}}{a^{6}}-3 \alpha_{2} \frac{c^{4}}{a^{12}}-5 \alpha_{3} \frac{c^{6}}{a^{18}}\right)- \\
-4 \pi \dot{\phi}^{2}\left(a^{2}+\kappa\left(\dot{a}^{2}+2 a \ddot{a}+4 a \dot{a}(\dot{\phi})^{-1} \ddot{\phi}\right)\right) .
\end{gathered}
$$

The equation of motion of the scalar field:

$$
a^{3} \ddot{\phi}+3 a^{2} \dot{a} \dot{\phi}-3 \kappa\left(\dot{a}^{3} \dot{\phi}+a \dot{a}(2 \dot{\phi} \ddot{a}+\dot{a} \ddot{\phi})\right)+V^{\prime}(\phi)=0 .
$$

We will make replacements to simplify these equations:

$$
\begin{gathered}
\tau=\ln a ; \quad \frac{d}{d t}=H \frac{d}{d \tau} . \\
\prime=\frac{d}{d \tau}, H=H(\tau), \phi=\phi(\tau) . \\
X(\tau)=H^{2}, \quad Y(\tau)=\kappa X .
\end{gathered}
$$

It is possible to choose two equivalent systems of the independent equations: (11) and (12); (11) and (13), which will result in only one equation. We will consider the system of the equations (11), (13), we will receive the equation on $X(\tau)$ :

$$
\begin{array}{r}
-\left\{8 \pi \kappa\left(c e^{15 \tau} m_{f}-c^{2} e^{12 \tau} \alpha_{1}-c^{4} e^{6 \tau} \alpha_{2}-c^{6} \alpha_{3}-e^{18 \tau} V\right)(1+9 X \kappa)+\right. \\
\left.+e^{18 \tau}\left(1-9 X \kappa+54 X^{2} \kappa^{2}\right)\right\} X^{\prime}+8 e^{18 \tau} \pi V\left(2-24 X \kappa+54 X^{2} \kappa^{2}\right)- \\
-2\left\{\left(4 c e^{15 \tau} m_{f} \pi+3 e^{18 \tau} X+8 c^{4} e^{6 \tau} \pi \alpha_{2}+16 c^{6} \pi \alpha_{3}\right) \times\right. \\
(-1+3 X \kappa)(-1+9 X \kappa)+4 e^{9 \tau} \sqrt{\pi} X \kappa \sqrt{-1+9 X \kappa} \times \\
\left.\sqrt{-3 e^{18 \tau} X-8 \pi\left(c e^{15 \tau} m_{f}-c^{2} e^{12 \tau} \alpha_{1}-c^{4} e^{6 \tau} \alpha_{2}-c^{6} \alpha_{3}-e^{18 \tau}\right) V} V^{\prime}\right\}=0 .
\end{array}
$$

We will notice that for $\alpha_{1}=\alpha_{2}=\alpha_{3}=m_{f}=0$ the equation is similar to the equation formulated in work Ref. 25. In the case of a lack of potential of a scalar field the equation (16) shapes into a simple form:

$$
\begin{array}{r}
2\left(3 e^{18 \tau} Y+4 e^{15 \tau} \tilde{m}_{f} \pi+8 e^{6 \tau} \pi \tilde{\alpha}_{2}+16 \pi \tilde{\alpha}_{3}\right)\left(1-12 Y+27 Y^{2}\right)+ \\
+\left(8 \pi(1+9 Y)\left(e^{15 \tau} \tilde{m}_{f}-e^{12 \tau} \tilde{\alpha}_{1}-e^{6 \tau} \tilde{\alpha}_{2}-\tilde{\alpha}_{3}\right)+\right. \\
\left.+e^{18 \tau}\left(1-9 Y+54 Y^{2}\right)\right) Y^{\prime}=0, \\
\tilde{m}_{f}=c \kappa m_{f}, \tilde{\alpha}_{1}=c^{2} \kappa \alpha_{1}, \tilde{\alpha}_{2}=c^{4} \kappa \alpha_{2}, \tilde{\alpha}_{3}=c^{6} \kappa \alpha_{3} .
\end{array}
$$

Thus the independent system of the field equations in lack of potential of a scalar field includes the equation (111) and the equation (17). 


\section{Resolution of cosmological constant problem due to coupled fermion}

The set of similar scalar models is considered in Ref. [17, but in our work the model is not only considered with the scalar, but also with the spinor field. We will contemplate solutions of the field equations (11), (16).

Equation (11) shows us that the existence of a special value for $\mathrm{H}$ follows, namely at $H=\frac{1}{3 \sqrt{\kappa}}$ coefficient at $\phi^{\prime 2}$ addresses in zero. Furthermore, it is shown that, in this case, there is a solution.

I) Solution $H=$ const $= \pm \sqrt{1 /(3 \kappa)}$.

In this case the potential of a scalar field plays a role of a cosmological constant $\Lambda$.

$$
\begin{gathered}
V(\phi)=\text { const }=\Lambda \\
a(t)=\beta e^{\frac{t}{\sqrt{3 \kappa}}}, \quad \beta-\text { const }, \\
\dot{\phi}^{2}=\Lambda-\frac{1}{8 \pi \kappa}+\frac{1}{\beta^{18}}\left(c^{6} e^{-\frac{6 \sqrt{3} t}{\sqrt{\kappa}}} \alpha_{3}+c^{4} e^{-\frac{4 \sqrt{3} t}{\sqrt{\kappa}}} \alpha_{2} \beta^{6}+c^{2} e^{-\frac{2 \sqrt{3} t}{\sqrt{\kappa}}} \alpha_{1} \beta^{12}-c e^{-\frac{\sqrt{3} t}{\sqrt{\kappa}}} m_{f} \beta^{15}\right),
\end{gathered}
$$

II) Solution $H=$ const $= \pm \sqrt{1 /(9 \kappa)}$.

$$
\begin{gathered}
a(t)=\beta e^{\frac{t}{\sqrt{9 \kappa}}}, \quad \beta, \gamma-\text { const } \\
\dot{\phi}^{2}=\gamma e^{\frac{2 t}{\sqrt{\kappa}}}-\frac{1}{4 \beta^{18}}\left(9 c^{6} e^{-\frac{6 t}{\sqrt{\kappa}}} \alpha_{3}+8 c^{4} e^{-\frac{4 t}{\sqrt{\kappa}}} \alpha_{2} \beta^{6}+6 c^{2} e^{-\frac{2 t}{\sqrt{\kappa}}} \alpha_{1} \beta^{12}-4 c e^{-\frac{t}{\sqrt{\kappa}}} m_{f} \beta^{15}\right), \\
V(\phi)=\frac{1}{24 \pi \kappa}-\frac{1}{\beta^{18}}\left(c^{6} e^{-\frac{6 t}{\sqrt{\kappa}}} \alpha_{3}+c^{4} e^{-\frac{4 t}{\sqrt{\kappa}}} \alpha_{2} \beta^{6}+c^{2} e^{-\frac{2 t}{\sqrt{\kappa}}} \alpha_{1} \beta^{12}-c e^{-\frac{t}{\sqrt{\kappa}}} m_{f} \beta^{15}\right) .
\end{gathered}
$$

We will now consider now solutions of the field equations in the absence of scalar potential $(V(\phi)=0)$, the equation (11) and (17). Asymptotic solutions are of greater interest to us in the equation (17) at small values $a(\tau \rightarrow-\infty)$ and big $a(\tau \rightarrow+\infty)$, and as transition from $\tau \rightarrow-\infty$ to $\tau \rightarrow+\infty$.

A) We will consider a case $\tau \rightarrow-\infty$, in this limit the equation (17) can be written down in the following way $\left(Y=\kappa X=\kappa H^{2}\right)$ :

$$
4(1-3 Y)(1-9 Y)-(1+9 Y) Y^{\prime}=0,
$$

For this equation there is a general solution:

$$
Y_{1,2}=\frac{1}{6}\left(2+3 \alpha e^{12 \tau} \pm \sqrt{\alpha e^{12 \tau}\left(9 \alpha e^{12 \tau}+8\right)}\right), \quad \alpha-\text { const } .
$$

The solution at $\tau \rightarrow-\infty$ takes the form

$$
Y_{1,2} \approx \frac{1}{3}\left(1 \pm \sqrt{2 \alpha} e^{6 \tau}\right) \rightarrow \frac{1}{3}
$$

For the equation (20) there are two partial solutions $Y=1 / 9, \quad Y=1 / 3$. The solution $Y=1 / 9$ - stable, $Y=1 / 3$ - unstable. There is a special value of $Y=-1 / 9$ 
for which $Y^{\prime}$ does not exist. At $\tau \rightarrow-\infty$ for $Y$ from an $(-\infty,-1 / 9)$ interval at approach for special value of $Y^{\prime}$ tends to $-\infty$, for $Y$ from an $(0,1 / 9)$ interval at approach for special value of $Y^{\prime}$ tends to $+\infty$, for $Y$ from interval of $(1 / 9,+\infty)$ the solution tends to $Y=1 / 9, a(t)=\alpha e^{\frac{t}{\sqrt{9 k}}}$. In the field of $Y \in(-\infty, 0)$ the main contribution to the equation gives the summands of the spinor field, generally summed with $\alpha_{3}$. If in the equation (20) we consider solutions of higher order of smallness, then the most important are summands with $\alpha_{2}, \alpha_{3}$ and the behavior of function $Y$ qualitatively doesn't change. Thus, if we put the potential of the spinor field equal to zero, we will receive a reversed diagram relative to the $O x$ axis. If we put $m_{f}, \alpha_{2}, \alpha_{3}=0$ we will receive singular solutions $Y=0, Y=-1 / 9$. Note that the results calculated in works Refs. 24, 25, in our case, are impossible, as in a limit at $\tau \rightarrow-\infty$ the spinor field renders considerable influence. If we exclude the spinor field, we will receive solutions given in the work above.

From the diagram (fig. 1) we can see that the accelerated expansion is observed. Thus, while comparing diagram (fig. 1 1 and fig. 2) we can see the prevalence (existence) of the summand spinor field, which causes faster expansion.

B) We will consider $\tau \rightarrow+\infty$ case. In this limit the equation (17) can be written down in the following way:

$$
6 Y(1-3 Y)(1-9 Y)+\left(1-9 Y+54 Y^{2}\right) Y^{\prime}=0,
$$

This equation is equivalent to the equation received in the work of Ref. 24 at $V(\phi)=0$, and, if we try to repeat the calculation it is possible to receive similar solutions.

As it is possible to find the general solution of this equation.

$$
\begin{gathered}
Y=\frac{2}{9}+\frac{2^{1 / 3} e^{6 \tau}}{9 F(\tau)}-\frac{2^{1 / 3} 3 \alpha}{F(\tau)}+\frac{2^{2 / 3} e^{-6 \tau} F(\tau)}{18}, \\
F(\tau)=\left(-2 e^{18 \tau}-81 \alpha e^{12 \tau}+9 \sqrt{\alpha e^{18 \tau}\left(8 e^{12 \tau}-27 \alpha e^{6 \tau}+972 \alpha^{2}\right)}\right)^{1 / 3},
\end{gathered}
$$

where $\alpha$ - an integration constant. If $\tau \rightarrow+\infty$, we have

$$
\begin{gathered}
F(\tau) \rightarrow-2^{1 / 3} e^{6 \tau}\left(1-3 \sqrt{2 \alpha} e^{-3 \tau}\right), \\
Y \rightarrow \frac{1}{3} \sqrt{2 \alpha} e^{-3 \tau}, H \rightarrow \frac{(2 \alpha)^{\frac{1}{4}}}{\sqrt{3 \kappa}} e^{-\frac{3 \tau}{2}} .
\end{gathered}
$$

For this equation there are three partial solutions $Y=0, Y=1 / 3, Y=1 / 9$. Solution $Y=0, Y=1 / 3$ - stable, $Y=1 / 9$ - unstable.

At $\tau \rightarrow+\infty$ for $Y$ from an $(-\infty, 1 / 9)$ interval solution aspire at big $\tau$ to $Y=0$, $a(t)=$ const, for $Y$ from an $(1 / 9,+\infty)$ interval solution aspire big $\tau$ to $Y=1 / 3$, $a(t) \rightarrow \alpha e^{\frac{t}{\sqrt{3 \kappa}}}$.

By considering an interval of positive values $\tau$ at $\tau \rightarrow+\infty$ we can observe a gradual weakening of the spinor field influence, and within the limit we receive the prevalence of the summands for a scalar field and the Einstein summand. 
June 22, $2021 \quad 7: 47 \quad$ WSPC/INSTRUCTION $\quad$ FILE

Article'Rybalov'Osetrin`Makarenkov

Note that in work Ref. 24 solutions, with additional asymptotic restrictions, for a conformal factor, in operation without the spinor field are received. In our case, however, the scalar field behaves differently, which is caused by the influence of the spinor field summands. As the existence of a spinor excludes a number of asymptotic solutions if we do not impose additional approximations (for example degree). From the diagram (fig. 21) we can see that the expansion accelerates and then we see deceleration. In a point 1.732 on an axis $O a(t)$ the curve breaks because further the function takes complex values.

\section{Conclusion}

The behavior of the cosmological model with the spinor field and non-minimally interacted scalar field has been considered. In the work a number of solutions addressing the exponential behavior of a large-scale factor for model with a scalar and spinor field have been calculated. That partial solution are solutions of $d S$ (deSitter), or solutions $a(t)=$ const. Asymptotic solutions are generally more difficult to comprehend and partial asymptotic solutions are the de Sitter's solutions or solutions $a(t)=$ const. If we impose additional conditions on the asymptotic equations (20), (23) it is possible to receive also the series solutions for a large-scale factor. The existence of the spinor field has an impact on evolution in the initial stage and further weakens its influence. Towards the latter stages of evolution, a scalar field and $R / 2$ term are very influential. Our following research will include the equivalent model with interaction of the scalar field and $f(R)$ gravitation.

\section{Acknowledgements.}

The work was partially supported by grant for leading Russian scientific schools, project No 88.2014.2 and grant of Ministry of Education and Science of Russian Federation, project No 867 .

\section{References}

1. S. Perlmutter et al., ApJ, 517, 565 (1999) arXiv: astro-ph/9812133.

2. S. Perlmutter et al., Nature 404, 955 (2000).

3. A.G. Riess et al., AJ, 116, 1009 (1998) arXiv: astro-ph/9805201.

4. C.L. Bennett et al., Astrophys.J.Suppl. 148, 1 (2003) arXiv: astro-ph/0302207.

5. C.B. Netterfield et al., Astrophys.J. 571, 604-614 (2002) arXiv: astro- ph/0104460.

6. N.W. Halverson et al., Ap.J. 568, 38 (2002), arXiv: astro-ph/0104489

7. A.H. Jaffe et al., Phys. Rev. Lett. 86, 3475 (2001), arXiv: astro-ph/0007333.

8. A.E. Lange et al., Phys. Rev. D 63, 042001 (2001).

9. A. Melchiorri et al., Ap.J. 563, L63 (2000), arXiv: astro-ph/9911445.

10. D.N. Spergel, et al. ApJS, 148, 175 (2003).

11. L. Verde et al., MNRAS, 335, 432 (2002).

12. I. Brevik, V.V. Obukhov, A.V. Timoshkin, TSPU Bulletin. 13, (128), p. 42-45, (2012) arXiv: gr-qc/1303.5669.

13. S. Nojiri and S.D. Odintsov, Phys.Lett. B599, 137 (2004) arXiv: astro-ph/0403622

14. S. Nojiri and S.D. Odintsov, Phys. Rev. D 68, 123512 (2003) arXiv: hep-th/0307288.

15. G. Allemandi, A. Borowiec, M. Francaviglia, and S.D. Odintsov, Phys. Rev. D 72, 063505 (2005) arXiv: gr-qc/0504057. 
June

22 ,

$2021 \quad 7: 47$ WSPC/INSTRUCTION

FILE

Article`Rybalov`Osetrin`Makarenkov

8 Osetrin K.E., Makarenko A.N., Rybalov Yu.A.

16. K. Bamba, A. Lopez-Revelles, R. Myrzakulov, S.D. Odintsov and L. Sebastiani. TSPU Bulletin. 13, (128), p. 22-27, (2012) arXiv: gr-qc/1301.3049.

17. K. Bamba, S. Capozziello, S. Nojiri and S.D. Odintsov, Astrophysics and Space Science, 342:155-228 (2012) arXiv: 1205.3421 [gr-qc].

18. S. Nojiri and S.D. Odintsov, Int.J.Geom.Meth.Mod.Phys. 4, 115-146 (2007) arXiv:hep-th/0601213

19. S. Nojiri and S.D. Odintsov, J. Phys. Conf. Ser. 66, 012005 (2007) arXiv: hep-th/0611071

20. S. Nojiri, S.D. Odintsov, Phys.Rept., 505, 59-144, (2011) arXiv: 1011.0544 [gr-qc].

21. K.E. Osetrin, Yu.A. Rybalov. Russian Physics Journal, 55, 1416 - 1424, (2013).

22. Yu.A. Rybalov, A.N. Makarenko, K.E. Osetrin. Astrophysics and Space Science, 349, N1, 561-566 (2014) arXiv: 1310.2505.

23. S.V. Sushkov, Phys. Rev. D80, 103505 (2009) arXiv: 0910.0980 [gr-qc].

24. E.N. Saridakis and S.V. Sushkov, Phys.Rev. D81, 083510 (2010) arXiv: 1002.3478 [gr-qc].

25. M.A. Skugoreva, S.V. Sushkov and A.V. Toporensky, Phys. Rev. D88, 083539 (2013) arXiv: 1306.5090 [gr-qc].

26. A.N. Makarenko, V.V. Obukhov, Entropy, 14, N7, 1140 (2012) arXiv: 1207.0589.

27. V.G. Bagrov, A.N. Makarenko, V.V. Obukhov, K.E. Osetrin, Grav.Cosmol.Suppl. 8, N2, 3-5 (2002).

28. A.N. Makarenko, V.V. Obukhov, Russ.Phys.J. 41, 1124-1133 (1998). 
June

22,

2021 7:47 WSPC/INSTRUCTION

FILE

Article'Rybalov ${ }^{*}$ Osetrin 'Makarenkov

(1)

COSMOLOGICAL MODELS WITH THE SPINOR AND NON-MINIMALLY INTERACTING SCALAR FIELD 9

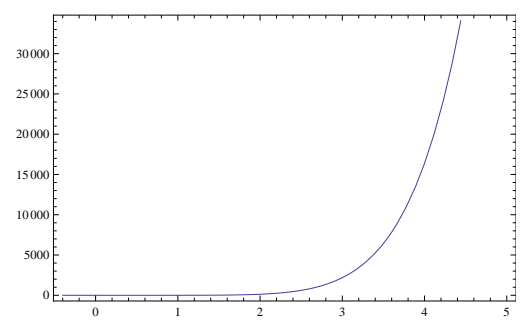

Fig. 1. Function $a^{\prime}(a), \tau \rightarrow-\infty, \kappa>0, \alpha=1$

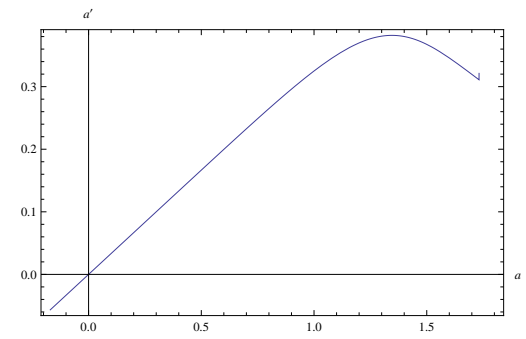

Fig. 2. Function $a^{\prime}(a), \tau \rightarrow+\infty, \kappa>0, \alpha=1$ 